\title{
Linx
}

LinX Revue des linguistes de l'université Paris X Nanterre

45 | 2001

Invariants et variables dans les langues. Études typologiques

\section{Passif et moyen dans les langues du pourtour méditerranéen}

\section{Ahmed Brahim}

\section{CpenEdition}

Journals

Édition électronique

URL : http://journals.openedition.org/linx/804

DOI : $10.4000 /$ linx.804

ISSN : 2118-9692

Éditeur

Presses universitaires de Paris Nanterre

Édition imprimée

Date de publication : 1 décembre 2001

Pagination : 107-116

ISSN : 0246-8743

Référence électronique

Ahmed Brahim, «Passif et moyen dans les langues du pourtour méditerranéen », Linx [En ligne],

45 | 2001, mis en ligne le 22 juin 2012, consulté le 01 mai 2019. URL : http://journals.openedition.org/ linx/804; DOI : 10.4000/linx.804

Ce document a été généré automatiquement le 1 mai 2019.

Département de Sciences du langage, Université Paris Ouest 


\title{
Passif et moyen dans les langues du pourtour méditerranéen
}

\author{
Ahmed Brahim
}

1 Cet exposé s'insère dans une recherche en cours en vue d'une typologie de la voix et de la diathèse dans les langues du Bassin méditerranéen. Il s'agit d'une recherche que j'essaie de mener dans une perspective théorique que j'ai déjà eu l'occasion de développer ailleurs et qui s'articule autour du concept de «relief actanciel » ou « relief argumental » fondé sur l'opposition entre « saillance » et « occultation » des participants du procès.

\section{Saillance et occultation}

2 Le degré de saillance ou d'occultation est conçu par rapport à un modèle de référence prototypique, celui des énoncés bi-actanciels où un terme source - disons un agent - est opposé à un terme cible - disons un patient - sur la base d'un ensemble de traits sémantiques et énonciatifs tels que le contrôle, l'humanitude, la définitude, la topicalité, etc.

3 1. 1. Du point de vue typologique, il peut être utile de concevoir cette opposition entre saillance et occultation comme opérant à trois niveaux, dont l'importance respective dépend de la manière dont les langues organisent leur système grammatical : le niveau actanciel proprement dit, c'est-à-dire celui des corrélations entre rôles sémantiques et relations grammaticales, le niveau ontologique - référentiel fondé sur la discernabilité intrinsèque des participants et enfin le niveau de la visée communicative concerné par la répartition de l'information dans l'énoncé.

4 Présents dans toutes les langues, ces niveaux sont hiérarchisés d'une manière spécifique qui diffère d'une langue à une autre, avec cependant certaines constantes qui autorisent à établir des tendances plus générales. C'est ainsi que les langues accusatives et les langues ergatives semblent organiser leur système grammatical essentiellement selon les paramètres du niveau 1 , alors qu'un système du type de celui des langues algonkiennes apparaît comme étant organisé principalement sur la base du niveau 2 et qu'un système 
du type des langues philippines et des langues qui leur ressemblent a plutôt tendance à privilégier le niveau 3.

5 Comment se présente la situation des langues méditerranéennes? Ce sont des langues qui, quoique à des degrés divers, accordent une importance primordiale à ce que j'ai appelé le niveau 1, celui des relations grammaticales dans leurs corrélations avec les rôles des participants. Et c'est par rapport aux phrases bi-actancielles caractérisées par une mise en œuvre maximale de la transitivité et de la saillance qu'il convient d'envisager celles qui s'écartent de ce modèle, c'est-à-dire qui présentent un degré plus ou moins élevé d'occultation, je veux parler en premier lieu des phrases «à voix marquée », mais aussi de celles où un phénomène assez comparable d'occultation peut avoir lieu sans sortir du cadre de la voix non marquée dite active.

6 1. 2. La plupart des linguistes travaillant dans le cadre de la syntaxe générale et de la typologie reconnaissent à toutes ces constructions un air de famille, beaucoup allant même jusqu'à les subsumer sous le label de "passif» tout en introduisant des différenciations en termes de proximité par rapport à un prototype. Mais il me semble qu'il faut relativiser l'importance de la voix et de la diathèse et user avec beaucoup de modération de la notion de passif prototypique en dehors des études portant sur des langues particulières qui possèdent un passif nettement délimité sur la base de critères essentiellement grammaticaux. Dans une perspective typologique, l'exploitation d'une catégorie grammaticale telle que celle du passif n'a pu, du reste, être rentable qu'au prix d'un glissement subreptice - mais bien réel - vers une acception sémanticoparagmatique. D'ailleurs, ce qui est "prototypique » pour l'analyste de telle ou telle langue ne l'est pas forcément pour son utilisateur, lequel peut, comme en (1) ci-dessous, passer dans le même énoncé d'une phrase active à sa reformulation passive (ou inversement), l'essentiel pour lui étant par exemple que l'agent du procès ne soit pas spécifié. Les formes peuvent varier et ce qu'on appelle le passif prototypique ne pas exister, la même faculté de paraphraser un énoncé par un autre se retrouve dans toutes les langues - v. par ex. (2) -, avec cependant une constante : l'occultation de l'agent.

\begin{tabular}{|l|l|l|l|}
\hline (1) & a. & $\begin{array}{l}\text { A la fin, on nous a répondu, il y a plus de vingt ans[...]Voilà la réponse qui nous a été donnée } \\
\text { (fr.parlé, cité par C. Blanche-Benveniste) }\end{array}$ \\
\hline & b. & Il ne douta pas qu'on ne le traitât aussi bien qu'il avait été traité (Voltaire,Candide) \\
\hline & & (2)ArT a. il-karahba t-serq-et-l-i & / b. il-karahba serq-u :-ha :-l-i \\
\hline & la-voiture RFX-voler+acp-3FS-à-moi & voler-acp-3MP-elle-à-moi \\
\hline & «On m'a voilé ma voiture » & \\
\hline
\end{tabular}

7 1. 3. C'est pour maintenir méthodologiquement distincts le niveau du signifiant et celui du signifié, les catégories grammaticales et les fonctions énonciatives, lesquelles en l'occurrence sont ce qui importe le plus, que j'ai proposé de donner à cet air de famille entre structures différentes (y compris sur le plan de la voix) le nom d'occultif.Si donc on admet que toutes ces constructions où l'agent est complètement passé sous silence ou relégué à la périphérie de la zone nucléaire des actants centraux, où la propriété même de l'agentivité est estompée et où l'élaboration des événements est à son degré le plus faible, 
si on admet, dis-je, que ces constructions relèvent d'une même fonction générale, alors il est possible, en intégrant le paramètre de la récession actancielle, d'établir pour cet occultif une classification assez schématique (et non exhaustive) telle que celle que l'on a en (3) :

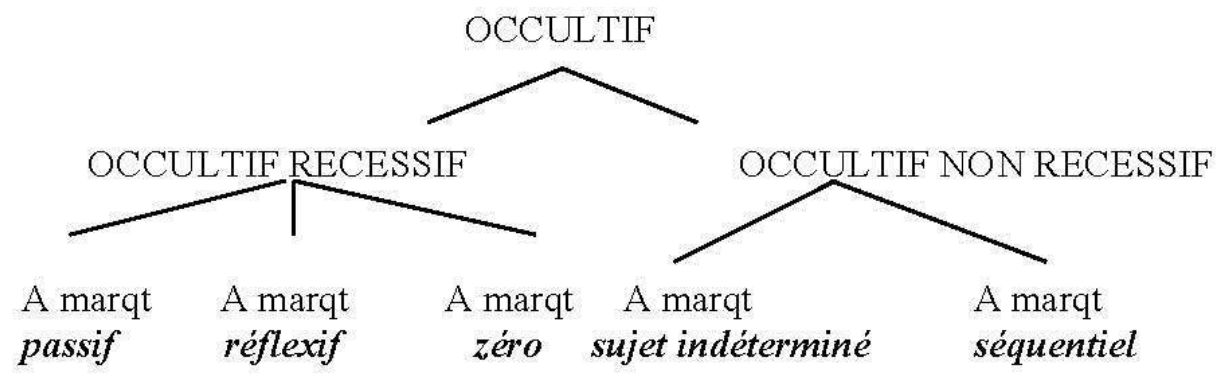

\section{L'occultif récessif à marquant passif}

Il convient tout d'abord de noter que ce qu'on appelle le " passif » est loin d'être, dans les langues du pourtour méditerranéen, le moyen le plus fréquent d'exprimer l'occultation de l'agent: il est soit inexistant, comme c'est le cas de tous les dialectes arabes, soit concurrencé ou même virtuellement supplanté par d'autres formes telles que les formes réflexives.

Même si, à l'intérieur de la catégorie du passif, la variante impersonnelle (i.e celle des verbes intransitifs) est marquée par rapport à la variante personnelle puisqu'il n'y a presque pas de langue, semble-t-il, qui posséderait un passif impersonnel sans avoir un passif personnel alors que la situation inverse se vérifie assez souvent - ce qui est d'ailleurs une des justifications de la notion de passif prototypique - il me parait cependant que c'est dans le passif impersonnel que se manifeste avec le plus de clarté la fonction d'occultation qui caractérise fondamentalement le passif en général. Si la forme impersonnelle ne constitue pas le "vrai» prototype du passif (encore que l'on soit parfois tenté de l'affirmer), elle semble du moins pouvoir en être l'archétype comme certaines études diachroniques (parmi lesquelles celle d'A. Rousseau sur l'indo-européen) tendent à le confirmer. C'est donc par la forme récessive des verbes intransitifs que je commencerai la présentation de l' « occultif récessif à marquant passif » dans les langues méditerranéennes.

11 2.1. Toutes les langues du Bassin méditerranéen qui possèdent un passif «canonique » semblent posséder également, quoique avec une fréquence inégale, un passif impersonnel. Le phénomène est fréquent en latin, en arabe classique et en hébreu biblique ainsi qu'en turc (4) mais plutôt rare dans les langues romanes (5), sporadique dans les langues slaves et en berbère, et quasi inexistant en grec et en albanais, toutes ces langues ayant développé d'autres modes d'expression du procès sans considération de l'agent.

Il est intéressant de noter que l'impersonnel en arabe classique, qui n'est d'ailleurs pas reconnu comme tel par les grammairiens, n'est possible pour les intransitifs qu'à la forme fu'ila dite mabni : li-l-majhu :l ((verbe)construit pour l'(agent)inconnu), ce qui confirme la 
fonction essentielle d'impersonnalisation de cette forme équivalente du passif des langues européennes. Dans tous les cas, un minimum d'ancrage est exigé qui prend la forme d'un circonstant quasi obligatoire. Quant à l'explicitation de l'agent sous la forme d'une expansion oblique, elle est en principe exclue puisque c'est la prédication de l'action verbale qui est mise au premier plan. Lorsqu'une telle expansion existe comme dans (4a), il faut y voir davantage l'expression d'une circonstance.

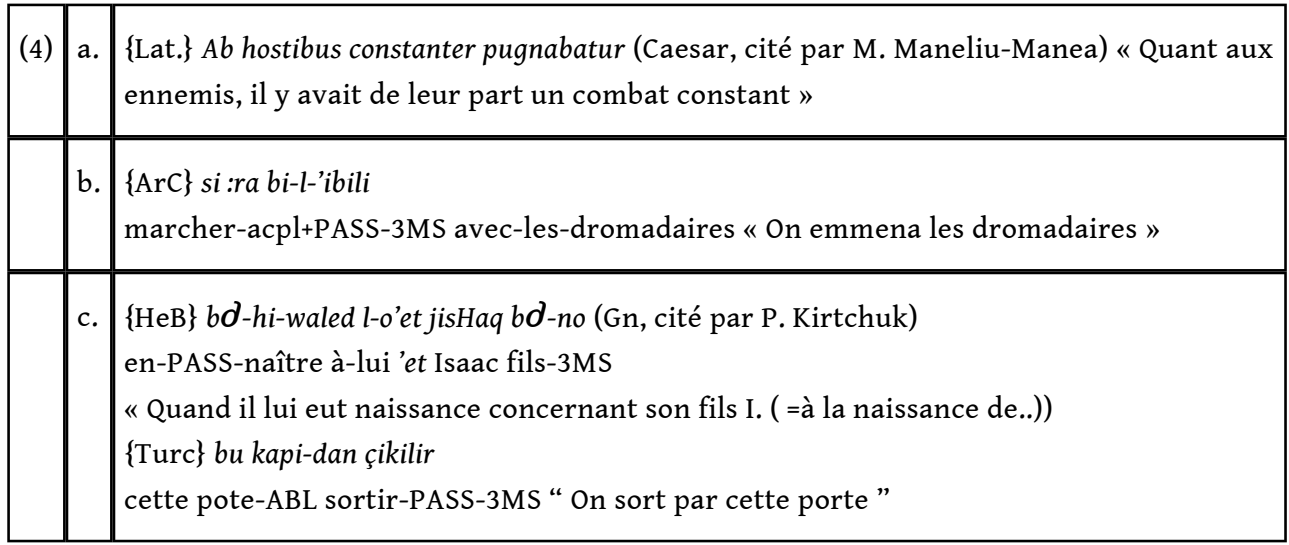

13 (5)\{Fr.\} Il a enfin été intervenu auprès de l'autorité de tutelle (N.Rivière) Dans les langues où ce type de passif impersonnel est d'un emploi assez courant, il s'applique aussi, comme on peut le constater en (6), aux verbes transitifs employés absolument :

\begin{tabular}{|l|l|l|}
\hline (6) & $\{$ ArC\} ya :'ayyuha :-lladi :na 'a :manu :'ida : nu :diya l-iS-Sala :ti...(Coran) \\
\hline & & quand appeler-PASS-MS pour-la-prière \\
\hline & \multirow{2}{*}{ « O vous qui croyez, quand on appellera à la prière... » } \\
\hline
\end{tabular}

ou accompagnés d'un complément interne (7), celui-ci étant, dans une langue comme l'arabe classique et standard, toujours disponible pour occuper la position sujet de la phrase passive canonique, c'est-à-dire celle d'un «substitut du sujet» d'après la terminologie grammaticale traditionnelle (8a). D'ailleurs, le complément interne est possible pour tous les verbes processifs, y compris les intransitifs, ce qui permet à ces derniers d'être le noyau de constructions passives du même type que les précédents (8b). Cette latitude morphosyntaxique qui fait que tout verbe processif peut, grâce au maSdar, se conformer de façon minimale au moule des passifs des verbes transitifs sans que l'action soit rapportée à un agent particulier, est sans doute la principale explication de l'inexistence dans la pensée grammaticale arabe, de la catégorie de l'impersonnel.

\begin{tabular}{|l|l|l|}
\hline (7) & $\{$ ArC Duriba Darb-an šadi:d-an \\
\hline & $\begin{array}{l}\text { fut-frappé un-frapper-ACC douloureux-ACC } \\
\text { «Il fut donné des coups durs / Il reçut des coups durs » }\end{array}$ \\
\hline
\end{tabular}




\begin{tabular}{|l|l|l|}
\hline (8) & a. & $\begin{array}{l}\text { Duriba Darb-un šadi :d-un } \\
\text { fut-frappé un-frapper-NOM douloureux-NOM } \\
\text { «Des coups douloureux furent donnés » }\end{array}$ \\
\hline b & $\begin{array}{l}\text { ruqiSa raqS-un jami :l-un } \\
\text { fut-dansé un-danser-NOM beau-NOM } \\
\text { "Il y eut une belle danse » }\end{array}$ \\
\hline
\end{tabular}

Plus répandue à travers les langues possédant un passif est la construction passive impersonnelle des verbes intransitifs « transitivés » au moyen d'une préposition (ou, si on préfère, transitifs indirects) ou régissant des compléments au datif. Ceux-ci sont fréquents en arabe classique, en turc ( $9 a$ et $b^{\prime}$ ) et dans les langues romanes (9c), où ils sont parfois soumis à des contraintes de temps (9c') et plus souvent, pour les langues autres que le français, remplacés par les formes réflexives :

\begin{tabular}{|c|c|c|c|}
\hline (9) & a & $\{$ Turc\}a. yeni kanun halka & anla-t-tir-il-di \\
\hline & & $\begin{array}{l}\text { nouvelle loi population-DAT expliquer-PASS- } \\
\text { Passé }\end{array}$ & (passif imp. du double factitit) \\
\hline & & \multicolumn{2}{|l|}{ «On a expliqué la nouvelle loi à la population» } \\
\hline & b. & ben kadin-la & konuş-tu-m \\
\hline & & \multicolumn{2}{|c|}{ je femme-avec parler-PASS-Passé+3S « Je parlais avec la femme» } \\
\hline & b'. & kadin-la & konuş-ul-du \\
\hline & & femme-avec parler-PASS-Passé+3S & $\begin{array}{l}\text { «Il y avait qq qui parlait avec la } \\
\text { femme; }\end{array}$ \\
\hline & & \multicolumn{2}{|l|}{ Ang. The woman was speaken with» } \\
\hline & & $\{$ Ita $\}$ & \\
\hline & c. & \multicolumn{2}{|l|}{ Fu proceduto ad un'autopsia } \\
\hline & $c^{\prime}$. & \multicolumn{2}{|l|}{ *Sarà proceduto / Si procederà ad un'autopsia } \\
\hline
\end{tabular}

Mais ce qui est plus intéressant encore, c'est que, contrairement à un idée reçue, le passif impersonnel est, dans plusieurs langues méditerranéennes, parfaitement compatible avec les verbes transitifs pourvus de leur complément d'objet direct (v.(10)), phénomène qui confirme encore la fonction d'impersonnalisation, ici primordiale même par rapport à la récession d'actance et à la promotion de l'objet :

\begin{tabular}{|l|l|l|l|}
\hline$(10)$ & a. & \{Ar.C\} wa y-uhraju la-hu & yawm-a-l-qiya:mati kita:b-an (Coran) \\
\hline
\end{tabular}




\begin{tabular}{|c|c|c|c|}
\hline & \multicolumn{3}{|c|}{ et 3MS-sortir-acpl à-lui jour-ACC la-levée livre-indéf-ACC } \\
\hline & \multicolumn{3}{|c|}{ «Et il lui sera sorti le Jour du jugement dernier un livre » } \\
\hline b. & \multicolumn{3}{|c|}{ Heb\} ye-haleq 'et ha-'ares (cité par I. Melcuk) } \\
\hline & \multicolumn{3}{|c|}{ 3MS-distribuer-PASS ACC la-terre } \\
\hline & \multicolumn{3}{|l|}{ «Est distribué la terre» } \\
\hline c. & \{Lat.\} Dicitur Homerum & caecum & fuisse \\
\hline & \multicolumn{3}{|c|}{ est-dit Homère-ACC aveugle-ACC-MS avoir été } \\
\hline & \multicolumn{3}{|c|}{ « Homère est dit voir été aveugle » } \\
\hline d. & \multicolumn{3}{|c|}{ \{Port.\} Vai ser transmitido hoje pela rádio os dois jogos de football } \\
\hline & \multicolumn{3}{|c|}{$\begin{array}{l}\text { «Il sera transmis aujourd'hui par la radio les deux matches de football " (usage non } \\
\text { standard) }\end{array}$} \\
\hline
\end{tabular}

2.2. Pour ce qui est du passif « promotionnel » où la position sujet est occupée par ce qui serait l'objet direct dans la phrase active, il peut lui-même être à la base d'une transformation impersonnelle dont l'effet est, dans une langue à ordre « canonique » SVO comme le français (11a-a'), de thématiser l'action verbale et de rhématiser le sujet passif, en faisant ainsi, du point de vue syntaxique, une sorte de complément de l'impersonnel (actant $\mathrm{H}$ dans la terminologie de G. Lazard). Ce genre de transformation semble plutôt peu répandu dans le domaine méditerranéen, où le même signifié est rendu par d'autres procédés (par ex. l'impersonnel du réflexif de sens passif). En arabe classique, l'invariabilité en nombre du verbe initial pourrait s'interpréter comme remplissant une fonction d'impersonnalisation initiale, mais cela n'est pas propre au passif puisqu'une phrase à verbe intransitif comme ja:'a rajulun se traduirait mieux par «Il est venu un homme » que par "Un homme est venu » (Idem pour qutila rajulun = «Il a été tué un homme »). En berbère, l'antéposition du verbe passif, associée à l'absence d'accord, peut être considérée comme une forme de passif impersonnel par transformation (v. 11b-b', exemple de Tamezret, Sud tunisien) :

\begin{tabular}{|c|c|c|c|}
\hline (11) & a. & \multicolumn{2}{|c|}{ \{Fr.\}Plus de mille exemplaires de ce livre ont été vendus } \\
\hline & $a^{\prime}$. & \multicolumn{2}{|c|}{ Il a été vendu plus de mille exemplaires de ce livre } \\
\hline & b. & \multicolumn{2}{|c|}{ Tam } akthar in alf $n$-il-kthab it-zinz-an \\
\hline & & \multicolumn{2}{|c|}{ 3-RFX-vendre+acp-PLU } \\
\hline & & \multicolumn{2}{|c|}{ «Plus de mille livres se sont vendus/ont été vendus» } \\
\hline & c. & $Y i-m-z i n z$ & alfn-ilkthab sig wa \\
\hline
\end{tabular}




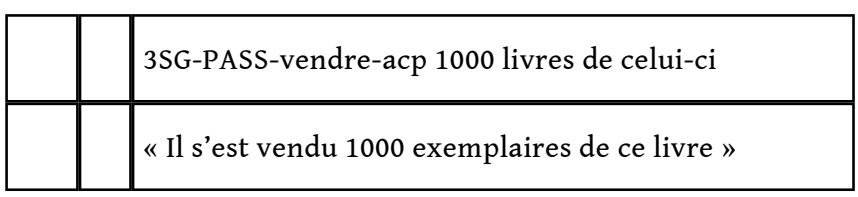

18 Ce type de construction semble exclure tout à fait la possibilité d'une expansion agentive, même lorsque celle-ci est d'un emploi normal dans les constructions passives personnelles correspondantes. Ces dernières, toujours marquées par rapport au passif non expansé, ne semblent tout à fait normales que dans les langues romanes et slaves, ainsi qu'en albanais et en grec. Elles sont plutôt exceptionnelles en arabe classique et en berbère; elles semblent devenir, sous l'influence des langues européennes, de plus en plus fréquentes en arabe littéral moderne et en turc :

\begin{tabular}{|c|c|c|}
\hline (12) & a. & \{Prov $\}$ Pau es estat/foguèt nafrat per Pèire (P.Sauzet) « Paul a été blessé par Pierre \\
\hline & b. & \{Bulg\} filmat e gledan ot zritelite « Le film est regardé par les spectateurs" \\
\hline & c. & $\{$ Turc\} kapi kapici tarafindan aç-il-di \\
\hline & & $\begin{array}{l}\text { porte portier de la part de ouvrir-PASS-Passé «La porte a été ouverte par le } \\
\text { concierge» }\end{array}$ \\
\hline & d. & $\{$ ArM\}'ustuqbila l-wazi :ru min tarafi/min qibali -rra'i :si \\
\hline & & $\begin{array}{l}\text { a-été-reçu le ministre de la part de le président "Le ministre a été reçu par le } \\
\text { président» }\end{array}$ \\
\hline & d'. & $\{$ ArC $\}$ 'urDi'a muHammadun min Hali :mata \\
\hline & & fut-allaité Mahomet de Halima « M. fut allaité par H » \\
\hline & e. & $\{$ Kabyl $\}$ y-Twadfr s wuSn (s.Chaker) \\
\hline & & il-a été suivi par chacal « Il a été suivi par un chacal» \\
\hline
\end{tabular}

19 Le plus souvent, la préposition (ou postposition) qui introduit cette expansion agentive a un sens spatial de provenance (Bg. ot, Gr. apó, Ar. min, Ber. s, etc.), ce qui rapproche les expansions concernées des circonstants. Loin d'être un argument contre la fonction occultive du passif, ces syntagmes la confirment, bien au contraire, et ce non seulement parce qu'ils équivalent à une mise à l'écart syntaxique mais aussi parce que, quand ils sont postposées, ils peuvent être énonciativement interprétées comme un mode de "désoccultation" rendu possible, ou plutôt préparé, par l'occultation préalable obtenue grâce au passif.

$\mathrm{Du}$ point de vue aspectuel, la plupart des langues concernées semblent distinguer la valeur processive du passif de sa valeur résultative-stative. Celles qui possèdent une forme périphrastique du passif à côté d'une forme synthétique (ou une forme adjective à côté d'une forme proprement verbale) ont tendance à spécialiser celle-ci dans l'expression du procès et celle-là dans l'expression de l'état : 


\begin{tabular}{|l|l|l|l|}
\hline (13) & a. & $\begin{array}{l}\text { \{Lat }\} \text { Gallia est omnis diuisa in partes très (cité par Touratier) « L'ensemble de la Gaule } \\
\text { est divisé en trois parties » }\end{array}$ \\
\hline & a'. & $\{$ Id. $\}$ Cum hic in duas partes diuideretur...(Id) « comme la rivière le coupait en deux... » \\
\hline & b. & $\{$ ArC $\}$ futiHa-lba :bu / al-ba :bu maftu :Hun \\
\hline & & a été ouverte la porte / la porte est ouverte \\
\hline \hline & c. & $\{$ Turc $\}$ kapi aç-il-di \\
\hline & & porte ouvrir-PASS-Passé & $/$ kapi aç-ik \\
\hline
\end{tabular}

21 La même tendance s'observe dans les langues slaves, où les formes périphrastiques sont, de ce point de vue, en distribution complémentaire avec les formes réflexives de sens passif. Parmi les langues romanes, le français se caractérise par l'ambiguïté de son passif périphrastique non expansé (la présence d'un complément d'agent peut orienter vers une lecture processive) ; à l'opposé, on a l'espagnol et l'italien distingue l'état du procès par le choix de l'auxiliaire, le premier en opposant estar à ser et le second essere à venire ou, plus rarement, andare lorsque ces auxiliaires sont à un temps simple :

\begin{tabular}{|l|l|ll|}
\hline$(14)$ & a. & $\{$ It. $\}$ a. La porta era chiusa “ La porte était fermée \\
\hline & b. & La porta veniva chiusa ogni sera alle sette dal guardiano \\
\hline & & «La porte était fermée tous les soirs à sept heures par le gardien » \\
\hline \hline & c. & *La casa è venuta costruita da moi padre /La casa è stata costruita da mio padre \\
\hline & & «La maison a été construite par mon père » \\
\hline
\end{tabular}

2.3. Concernant les verbes trivalents (verbes de don, etc.), seules quelques langues les tournent au passif avec promotion du « donataire » : il s'agit précisément de celles (arabe classique, hébreu, latin) qui peuvent avoir à l'actif deux compléments directs à l'accusatif :

\begin{tabular}{|c|c|c|c|}
\hline$(14)$ & a $\{$ ArC $\}$ 'u :tiya & ban-u :'isra :'i :l-a & -lkita :ba (Coran) \\
\hline & \multicolumn{3}{|c|}{ faire-venir-PASS-acp-3MS fils-PL-NOM Israël-GEN le-livre-ACC } \\
\hline & « Les enfar & arent le livre sa & \\
\hline
\end{tabular}


tres langues recourent à des paraphrases diverses (réflexif factitif en français, thématisation du donataire avec le passif de l'objet donné en berbère, antéposition du donataire avec maintien de sa marque casuelle en turc, etc ;) :

\begin{tabular}{|l|l|l|}
\hline (15) & a. & \{Fr.\} Pierre s'est fait/vu donner un livre \\
\hline \hline & b. & $\{$ Tam.\} Ali yimm-uš-aš ilkthab (sig Mansur) \\
\hline & & Ali, il lui a été donné un livre (par Mansour) \\
\hline & c. & $\{$ Turc\} Hasan'a Ali trafindan bir kitap ver-il-di \\
\hline & & A Hassan par Ali un livre a été donné \\
\hline
\end{tabular}

\section{L'occultif à marquant réflexif}

Ce que l'on peut remarquer d'emblée, c'est que l'aire d'emploi du passif est, dans les langues méditerranéennes, beaucoup moins étendue que celle de ce qu'il est permis d'englober sous l'appellation générale de "réflexif": cela est assez évident dans les langues slaves et la majorité des langues romanes mais aussi ailleurs. En effet, si on définit schématiquement la réflexivité à la fois sémantiquement par l'indifférenciation entre agent et non agent dans le sujet et morphosyntaxiquement par la présence d'une marque de récession actancielle, alors on peut appeler réflexives les formes traditionnellement non considérées comme telles, je veux parler du moyen grec, des déverbatifs affixés des divers parlers arabes, ainsi que de ce que les berbérisants appellent les « verbes réfléchis ».

A ce propos, il me paraît nécessaire d'établir une distinction nette entre réfléchi et réflexif (ou, en anglais, entre reflective et reflexive ), celui-ci étant une sorte de détransitivant alors que celui-là occupe une position argumentale et remplit une fonction syntaxique distincte. Notons qu'on pourrait tout aussi bien appeler «moyennes» les formes appelées ici réflexives, mais il est préférable de spécialiser le terme de moyen dans la désignation des faits de diathèse, conçus comme différents des phénomènes concernant la voix, c'est-àdire de donner au mot moyen une extension telle qu'il puisse englober les cas où le verbe n'est pas marqué (comme dans La branche casse vs La branche se casse vs La branche est cassée).

Cette distinction entre réfléchi et réflexif est grammaticalisée dans certaines langues ( $\mathrm{v}$ (16)) alors que, dans d'autres, on a en synchronie deux morphèmes homonymes (v.17)) :

\begin{tabular}{|c|c|c|c|c|c|}
\hline \multirow[t]{2}{*}{$(16)$} & \multirow[t]{2}{*}{ a. } & \multicolumn{2}{|c|}{ Turc } Ahmet kendi-ni yika-di & \multicolumn{2}{|c|}{ /a'. Ahmet yIka-n-di } \\
\hline & & A & RFC-ACC laver-Passé & A. & laver-RFX-Passé « Ahmed se lave » \\
\hline & b. & \multicolumn{2}{|c|}{ ArT } 'li :jraH ru :H-u & \multicolumn{2}{|c|}{ / b'. 'li: $t$-[ [- } \\
\hline & & \multicolumn{2}{|c|}{ A a-blessé RFC( =âme)-3MS } & A R & blessé « Ali s'est blessé » \\
\hline
\end{tabular}




\begin{tabular}{|c|c|c|c|c|}
\hline & c. & \{Bulg\} Deteto gleda sebe si / c'. se gleda & $v$ & ogledaloto \\
\hline & & enfant-le regarde RFC & \multicolumn{2}{|c|}{ RFX-regarde dans la glace } \\
\hline & & \multicolumn{3}{|c|}{ «L'enfant le regarde » / "L'enfant se regarde dans le miroir» } \\
\hline & c". & \multicolumn{2}{|l|}{ Deteto se sărdi } & /*sărdi sebe si \\
\hline & d. & $\{$ Tam.\}'li i-raqib imani-s & ig & thimrayith \\
\hline & & \multicolumn{3}{|c|}{ Ali regarde RFC-3MS dans la glace « Ali se regarde dans la glace } \\
\hline & d'. & $y i-t-m a r a$ & waHdis & \\
\hline & & \multicolumn{3}{|c|}{ la porte 3-RFX-ouvre seule « La porte s'ouvre d'elle-même » } \\
\hline (17) $\{\mathrm{Fr}\}$. & a. & \multicolumn{3}{|l|}{ Pierre se voit / a'. Pierre se réveille } \\
\hline & b. & \multicolumn{3}{|c|}{ Pierre ne voit que lui-même/ b'. 'Pierre ne réveille que lui-même } \\
\hline & & \multicolumn{3}{|c|}{ C'est lui-même que P voit / c'.* C'est lui-même que P réveille } \\
\hline & c. & \multicolumn{3}{|l|}{ ??Pierre ne lave que lui-même } \\
\hline (18) $\{$ It. $\}$ & a. & \multicolumn{3}{|c|}{ Maria (se) ha tagliata se stessa / b. Maria si è tagliata } \\
\hline
\end{tabular}

Même dans les cas d'homonymie, la différence structurelle entre réfléchi et réflexif peut être mise au jour grâce à des tests tels que la négation restrictive (18b-b') et le clivage (18c-c'), qui montrent l'autonomie insuffisante de se par rapport à réveiller, même si celuici est commutable dans les deux cas avec un clitique non coréférent (Pierre le voit / le réveille). L'anomalie de (18 b'-c') reflète l'anomalie sémantique qu'il y a à attribuer deux participants à un procès qui est nettement intransitif (i. e monovalent). Il en est de même dans (19), où on peut opposer plus clairement le réflexif clitique de (19b) au réfléchi emphatique de (19a). Sur le plan sémantique, le sujet de (19a) est à interpréter comme un agent, alors que celui de (19b) est plutôt neutre du point de vue de l'agentivité, celle-ci étant seulement impliquée par le trait d'humanitude. C'est seulement dans (19a) que l'on a une opposition entre agent et patient, lesquels sont conceptuellement différenciés bien que référentiellement identiques. A l'inverse, l'absence d'une telle différenciation en (19b) apparaît comme étant quelque peu paradoxale étant donné que le verbe tagliare implique une action typiquement orientée vers l'extérieur.. Mais cette implication d'agentivité semble tenir du comportement syntaxique des clitiques réflexifs - réfléchis propre aux langues romanes. Dans les langues où le signifiant du réflexif est distinct de celui du réfléchi, cette implication d'agentivité se révèle être, en effet, beaucoup plus ténue (v. (17b')).

$28 \mathrm{Si}$, au lieu d'un procès tourné vers l'extérieur, on prend un procès typiquement - et culturellement - tourné vers l'ego (se laver, se raser, etc.), on voit que le clitique se réflexif du français est encore moins distinct de son alter ego réfléchi, ce qui est d'ailleurs répercuté dans les grammaires, qui l'analysent traditionnellement comme un COD, c'està-dire comme un "vrai » réfléchi. Même en bulgare, le test de remplacement de se par 
sebe si ne donne pas lieu à une quelconque agrammaticalité (20a), comme c'est le cas par exemple pour sărdi (se fâcher) en (17c"). Dans cette langue, il est également possible de faire commuter se avec le pronom non coréférent go (20b) :

\begin{tabular}{|l|l|l|}
\hline (20) \{Bulg.\} & a. & Deteto se mie / Deteto mie sebe si \\
\hline \hline & & «L'enfant se lave » \\
\hline & b. & Deteto go mie « L'enfant le lave » \\
\hline
\end{tabular}

Cependant, il faut souligner ici que, dans le cas où l'identification de l'objet - patient avec le sujet - agent est exprimée par sebe si, il y a un effet de contraste par lequel l'objet est opposé à tout autre objet non spécifié : l'opposition agent / patient est aussi nette qu'elle le serait dans une phrase transitive active, tandis que dans se mie et se laver, l'action de «laver» est interne au domaine du sujet - agent. Là encore, le test de la négation restrictive peut être assez discriminant (v.(18d)) et confirmer que la signification est en l'occurrence celle d'un moyen, non celle d'un vrai réfléchi : ? ?Pierre ne lave que lui-même.

Il est à noter que cette particularité sémantique pourrait être expliquée, comme j'ai proposé de le faire ailleurs (Brahim, 2001) en des termes proches de ceux de Culioli, dans la mesure où l'on a une configuration à deux places dont la première n'est pas spécifiée quant à l'agentivité : par une série d'opérations, une relation est obtenue où il y a coïncidence entre un terme source et un terme cible, c'est-à-dire une sorte de boucle. L'actualisation interprétative du rôle sémantique du sujet est le résultat d'une inférence à partir du sémantisme du verbe et des caractéristiques ontologiques inhérentes $d u$ référent (animé ou inanimé, etc.).

31 C'est ainsi que le faible degré de transitivité dans une telle relation peut conduire à un degré plus ou moins élevé d'occultation valencielle ou récessivité actancielle, consistant en une opposition ou un continuum entre implication et non-implication d'un agent externe au référent du sujet grammatical, que l'on peut illustrer, pour le français, par les exemples donnés en (21) :

\begin{tabular}{|l|l|l|}
\hline$(20)\{\mathrm{Fr}\}$. & a. & La porte s'est ouverte (toute seule) / a'. La porte a été ouverte \\
\hline & b. & La porte s'ouvre facilement (il y suffit d'un peu de vent) \\
\hline & c. & La porte s'ouvre facilement (il suffit de la pousser) \\
\hline
\end{tabular}

Ainsi l'exemple (21a) peut être opposé à (21a'), où il y a clairement un agent impliqué : la forme réflexive de (21a) ajoutée à ses caractéristiques aspectuelles et temporelles conduit à l'interprétation de son verbe comme étant un « moyen spontané " pouvant être spécifié grâce à une expansion attributive (prédication seconde) comme toute seule. D'un autre côté, les exemples (21b) et (21c) auront respectivement une interprétation médio-passive et passive : ici, le caractère non agentif du sujet est induit du fait qu'il s'agit d'un non animé. 


\begin{tabular}{|l|l|l|l|l|}
\hline a. & le & réflexif & "subjectif», & i.e « agentif» \\
\hline b. & - & - & "passif» & \\
\hline c. & - & - & « impersonnel & \\
\hline d. & - & - & "non agentif & \\
\hline
\end{tabular}
début de cet exposé l'occultif non récessif (phrases actives dont le sujet est On ou tout autre forme de $3^{\text {ème }}$ personne, surtout au pluriel, stratégie extrêmement fréquente dans les parlers arabes et berbères et possible presque partout ailleurs). Il en est de même de ce que j'ai appelé l'occultif non récessif à marquant séquentiel qui constitue un moyen de thématisation fort commode permettant par exemple d'obtenir des équivalents de phrases passives avec complément d'agent ou des passifs du «tiers actant » des verbes trivalents.

38 Ce sont là des structures dont l'importance est trop souvent « occultée » mais que je n'ai pas le temps de d'aborder ici, pas plus que les verbes à double orientation (ou occultif récessif sans marquant) si essentiels dans le domaine berbère. 


\section{BIBLIOGRAPHIE}

BENVENISTE, E. (1966) : Problèmes de linguistique générale, 1, Paris : Gallimard.

BRAHIM, A. (1995) : Da :hiratu l-Hajbi fi : bina :'i l-fi'li wa l-jumlati fi : l- 'arabiyyati wa luGa :tin 'uhra : « Le phénomène de l'occultation dans la structure du verbe et de la phrase en arabe et dans d'autres langues », in Hawliyya :t al-ja :mi'a -ttu :nisiyya, $\mathrm{n}^{\circ} 36$.

(1996) : L'occultif : hypothèse pour un traitement trans-linguistique du passif et des constructions apparentées, Tunis : Publications de l'ATL.

(2000) : Relief actanciel et diathèse verbale, in L. Shoesler (éd) : Le passif, Copenhague : Museum Tusculanum Press.

(2001) : Some Aspects of Reflexive-Marked Occultive, in T. Stolz (ed) : Proceedings of the Workshop on "Languages of the Mediterranean Area, Brême : STUF.

CUlioli, A. (1990) : Pour une linguistique de l'énonciation : opérations et représentations, Paris : Ophrys.

DESCLÉS, J. P., GUENTCHÉVA, Z. \& SHAUMYAN, S. (1986) : Theoretical Aspects of Reflexivization in the Framework of Applicative Grammar, in Linguisticae Investigationes, X/1, Paris : Klincksieck.

FICI Giusti, F. (1998) : Diathèse et voix marquée dans les langues d'Europe, in J. Feuillet (éd) : Actance et valence dans les langues d'Europe, Berlin - New York : Mouton de Gruyter.

GALAND, L. ( 1987) : Distribution des rôles dans l'énoncé verbal en berbère, in Actances 3, Paris, :RIVALC.

GUENTCHÉVA, Z. (1989) : A propos des constructions réflexives en bulgare, in Actances 4, Paris : RIVALC.

KEENAN, E. (1985) : Passive in the World's Languages, in T. Shopen (ed) : Language Typology and Syntactic Description, 1, Cambridge : Cambridge University Press.

KEMMER, S. (1993) : The Middle Voice, Amsterdam - Philadelphia : J. Benjamins.

KLAIMAN, M. (1991) : Grammatical Voice, Cambridge.

KHRAKovsky, V. (1973) : « Passive Constructions (Definition, Calculus, Typology and Meaning) », in J. Kiefer (ed) : Trends in Soviet Theoretical Linguistics : Foundations of Language, Supplementary Series, 18, The Hahue : Dorrdrecht.

LAZARD, G. (1986) : Formes et fonctions du passif et de l'antipassif, in Actances 2, Paris : RIVALC.

(1995) : L'actance, Paris : Puf.

RIVIÈRE, N. (2000) : Le pronominal face à l'actif et au apassif, in L. Shoesler (éd) : Le passif, Copenhague : Museum Tusculanum Press.

SHIBATANI, M ; (1985) : Passive and Related Constructions, in Language, 61 / 4

TESNIÈRE, L (1959) : Eléments de syntaxe structurale, Paris : Klincksieck. 
AUTEUR

AHMED BRAHIM 